\title{
Complications rate and risk factors in unplanned reoperation of degenerative lumbar spondylolisthesis in geriatric patients: a retrospective single-center cohort study of 33 patients
}

\section{Weiyang Zhong}

Chongqing Medical University First Affiliated Hospital

\section{Xinjie Liang}

Chongqing Medical University First Affiliated Hospital

Xiaoji Luo ( $\nabla$ cy2982@163.com )

Chongqing Medical University First Affiliated Hospital

\section{Tianji Huang}

Chongqing Medical University First Affiliated Hospital

Zhengxue Quan

Chongqing Medical University First Affiliated Hospital

\section{Research article}

Keywords: degenerative lumbar spondylolisthesis, unplanned operation, risk factor, geriatric patients

Posted Date: December 15th, 2019

DOI: https://doi.org/10.21203/rs.2.18807/v1

License: (9) This work is licensed under a Creative Commons Attribution 4.0 International License. Read Full License

Version of Record: A version of this preprint was published on August 24th, 2020. See the published version at https://doi.org/10.1186/s12877-020-01717-2. 


\section{Abstract}

Background: The study aimed to investigate the complications rate and risk factors in unplanned reoperation among geriatric patients who underwent posterior lumbar fusion(PLF) for degenerative lumbar spondylolisthesis(DLS).

Methods: 1100 DLS patients who were older than 60 years were reviewed from January 2006 to December 2016 and 33 patients underwent unplanned reoperations who were analyzed and divided to two groups(Group A: posteriolateral fusion, Group B: intervertebral fusion). Gender, body mass index (BMI), radiographic data and clinical outcomes data were analyzed to evaluate complications rate and the risk factors for unplanned reoperations.

Results: 33 patients were performed by the unplanned reoperations(3\%).The patients were followed up for an average of $4.20 \pm 2.25$ years (Group A)and $4.32 \pm 2.54$ years(Group B)without significant difference. The significant difference was found in mean age, levels involved levels, hospital stay, surgery time, blood loss between the groups. The causes of unplanned operation were: wound infection, screw misplacement, neurological deficit, nonunion, screw fracture which were significant except the wound infection between the groups. Higher BMI (obesity), diabetes mellitus(DM), more bleeding and gender(female) were the risk factors of the complications. The cases of the screw misplacement, neurological deficit, nonunion and screw fracture in group A were more significantly than in group B.

Conclusion: The patients of higher BMI, DM, age, posteriolateral fusion, female predicted higher incidence of unplanned reoperations. The spine surgeons maybe need to pay more attention in their preoperative training and in improving surgical technique which could reduce the reoperation rate.

\section{Background}

As the arrival of the aging population, the incidence of degenerative lumbar spondylolisthesis(DLS) and its surgical treatments also get more and more attention, because the rapid progress of anesthesiology and surgical instruments lead to active surgical management in geriatric patients[1]. However, preventing revision spinal surgery is still a matter of cardinal significance for the spine surgeons and their patients. Unplanned reoperation means an unexpected event, a status of persistent symptoms, deterioration of the previous potential diseases, or complications related to the primary operation[2-3]. A better understanding of the complications rate and risk factors of unplanned reoperation could help improve the surgical outcomes and prognosis. It was reported that the cumulative reoperation rate in lumbar degenerative diseases was $4.7 \%$ at 3 months, $6.1 \%$ at 1 year, $8.5 \%$ at 2 years, $15.2 \%$ at 3 years, $17.7 \%$ at 5 years and $23.3 \%$ (38/163 patients) at the final follow-up[4-8]. Reducing rate of lumbar surgery revision is also vital because of endings of DLS reoperation for may be worse than the the initial surgical management results. 
Therefore, the study aimed to investigate the incidence of unplanned reoperation in geriatric patients performed surgically for DLS and to analysis the complications rate and risk factors.

\section{Methods}

\section{Patient selection}

The study was approved by the institutional review board of ourhospital. 1100 patients were restropectively reviewed and the unexpected reasons for the unplanned reoperation were recorded and assessed. Between January 2006 and December 2016, 33 reoperation patients with DLS, aged more than 60 years old were enrolled.

The inclusion criteria: one-level or two-level DLS requiring surgical treatment again. All the surgeries were performed of posterior lumbar fusion including posterolateral fusion and intervertebral fusion. Unplanned surgery was defined as receiving the reoperation in operation room after the primary surgery. The primary and revision surgery were all performed by the same senior surgeons team. The exclusion criteria: the patients suffered the lumbar disc herniation, or lumbar spinal stenosis, or lumbar vertebral fractures, spondylitis and tumors.

Patients' demographic data, including the primary surgical procedure and revision surgery information were recorded in our study. The reasons for the unplanned surgery were assessed and the reoperation rates of DLS were calculated.

\section{Statistical analysis}

All statistic data were analyzed with SPSS version 22.0 statistical software(SPSS, Inc., Chicago,IL,USA). The data were analyzed by Student's t-test for continuous variables and Chi square test for categorical variables. Values of $\mathrm{P}<0.05$ were regarded statistically significant. For all regression models, a $\mathrm{p}$ value less than 0.05 with a confidence interval $(\mathrm{Cl})$ of $95 \%$ was considered significant.

\section{Results}

33 patients were performed by the unplanned reoperations(3\%). The patients were followed up for an average of $4.20 \pm 2.25$ years (Group A) and $4.32 \pm 2.54$ years(Group B) without significant difference. The significant difference was found in mean age, levels involved levels, hospital stay, surgery time, blood loss between the groups (table 1). The causes of unplanned operation were: wound infection, screw misplacement(Figure 1), neurological deficit, nonunion(Figure 3,4), screw fracture(Figure 2) which were significant except the wound infection between the groups. The cases of the screw misplacement, neurological deficit, nonunion and screw fracture in group A were more significantly than in group $B$.

Table 1 Comparison of the baseline data 


\begin{tabular}{llll} 
& Posteriolateral fusion & Intervertebral fusion & P value \\
\hline Patients $\llbracket \mathbf{n} \rrbracket$ & 24 & 9 & $<0.0001$ \\
Males/females & $9 / 15$ & $3 / 6$ & \\
\hline Mean age(y) & $72.80 \pm 11.7$ & $71.90 \pm 10.7$ & 0.583 \\
\hline Follow-up(y) & $4.20 \pm 2.25$ & $4.32 \pm 2.54$ & 0.654 \\
\hline Involved levels & & & \\
L4-5 & 18 & 5 & $<0.0001$ \\
L5-S1 & 6 & 4 & $<0.0001$ \\
\hline Hospital stay (days) & $12.55 \pm 3.35$ & $15.43 \pm 5.20$ & $<0.0001$ \\
\hline Surgery time (minutes) & $100.50 \pm 30.50$ & $15.43 \pm 5.20$ & $<0.0001$ \\
\hline Blood loss (ml) & $150.65 \pm 35.45$ & $230.90 \pm 101.50$ & $<0.0001$ \\
\hline Wound infection & 4 & 3 & 0.730 \\
\hline Screw misplacement & 7 & 3 & $<0.0001$ \\
\hline Neurological deficit & 5 & 1 & $<0.0001$ \\
\hline Nonunion & 4 & 1 & $<0.0001$ \\
\hline Screw fracture & 4 & 1 & $<0.0001$
\end{tabular}

The wound infection were revised for debridement surgery without removing implants, who were treated with antibiotics sensitive to the bacterial. The pedicle screws displacement were detected on thepostoperative $\mathrm{X}$-ray from those patients manifested leg pain or persistent sciatica after the primary surgery. All of the screws were corrected immediately and the leg pain improved after the reoperation and the recovered to normal at the final follow-up.

\section{Risk factors analysis}

Based on the previous documents, the potential factors were selected and assessed for analyzing risk factors, the following data: age, gender, body mass index(BMI), fusion methods of surgery fusion. Higher $\mathrm{BMI}$, diabetes mellitus(DM), more bleeding and women predicted higher occurrence of wound infection(Table 2). Different fusion methods, female predicted the development of nonunion and screw fracture.

Table 2 Risk analysis for wound infection 
Valuables OR P value 95\% confidence interval

\begin{tabular}{lllll}
\hline BMI (obesity) & 4.42 & 0.01 & 1.5 & 14.06 \\
\hline Gender(female) & 3.82 & 0.015 & 1.05 & 9.73 \\
\hline Diabetes mellitus & 3.08 & 0.025 & 0.78 & 7.85 \\
\hline Age & 3.65 & 0.02 & 0.96 & 1.45 \\
\hline Fusion method & 4.02 & 0.010 & 2.01 & 16.21 \\
\hline More Bleeding & 3.01 & 0.03 & 0.65 & 7.21
\end{tabular}

BMI: body mass index.

\section{Discussion}

With the increase of the elderly population in China, more and more aged patients with degenerative lumbar diseases(DLD) require surgical treatment. DLS is a type of spondylolisthesis with an intactneural arch. The treatment objectives of surgery contain neural decompression, the motion segment stabilization, intervertebral disc heightreconstruction and sagittal balance restoration[1-2][9-12]. The spondylolytic segment stabilization is importantly relied on fusion through a anterior, a posterior or combined approach[12-13]. The PLF technique has gained reliability and popularity for DLS. Posterolateral fusion or intervertebral interbody fusion immediately reconstruct a fast biomechanically stable spine, thereby increasing the chance of fusion[14-16]. However, in geriatric patients, it is a challenge to perform spine surgery because of the increasing presence of medical diseases and surgical complications[16]. Furthermore, the reoperation is an unplanned event for the patients, their family and surgeons, which brings more perioperative complications, including deaths and potential medical risks or medical disputes. Finding the risk factors for surgeons are important to make better decision preoeperation and to evaluate the surgery procedures for avoiding the unplanned reoperations.

Several previous studies reported reoperation following an primary lumbar surgery for degenerative conditions and indicated that the revision rates were $14.0 \%$ in the 1997 to 2000 cohort and $12.4 \%$ in the 1990 to 1993 cohort[17]. Ghogawala demonstrated that the re-operation rate after only decompression for DLS at 1-year postoperatively was $15 \%[18]$ while Blumenthal reported $37.5 \%$ at a mean follow-up of 3.6 years[19]. The reoperation rate occurred in the present study nearly as the same as in the previous studies: the reoperation rate for only decompression at 1-year follow-up was $10.8 \%$ and the rate increased to $29.7 \%$ at 5 -year postoperatively and to $33.4 \%$ at the final follow-up[20-21]. Another report demonstrated the cumulative reoperation rate was $6.1 \%$ at 1 year, $8.5 \%$ at 2 years, $15.2 \%$ at 3 years, $17.7 \%$ at 5 years, and $23.3 \%$ at the final follow-up[22]. Different from the studies mentioned above, the reoperation rate of 
DLS was 3.0\% in our study, which was lower than those previous studies. The reason for the lower reoperation rate in our study may be that we just reported the reoperation during patients of one-level or two-level DLS.

Elderly patients have commonly been considered to be at a higher risk of postoperative complications of DLS than younger patients. It was documented an approximate $80 \%$ of complications rate who were performed surgically for DLS[23-27]. In the study of Okuda, postoperative complications were found in $16 \%$ of elderly patients after PLIF with pedicle screw placement[28]. In our series, the major reasons for the reoperation were wound infection, screw misplacement, neurologic deficit, nonunion, screw fracture, which were similar to previous reports. Therefore, the better understanding of the complications, risk factors and unplanned reoperations rate is helpful to improve the surgical outcomes.

In our study, higher BMI , DM, more bleeding and women predicted the occurrence of wound infection, which other studies have previously reported. Postoperative wound deep infection will significantly impact the surgical outcomes of DLS and have an important influence on surgical plan which need the revision surgery, removing the implants if necessary. It also increased the cost of the hospitalization and nursing. And the spine surgeon perhaps pay more attention to improve the surgical training and need more help of multiple disciplinary team(MDT).

Another common reason for unplanned reoperation was the screw displacement in the study. Complications related to screw displacement in spinal surgery are more common and often result in spinal cord and nerve root injury as well as dural lesion which require revision surgery immediately. Fortunately, all neurological defects of the patients after reoperation alleviated and returned to normal at the final follow-up. More spine courses or navigation system could help reduce the rate of screw displacement. Also we found that the patients who underwent posteriolateral fusion procedure which had higher incidence of nonunion and higher rate of unplanned reoperation. It showed that detailed preoperative plan and strict follow-up could work for patients with DLS when indicated for surgical treatment.

\section{Conclusion}

The unplanned reoperations rate in geriatric patients surgically performed for DLS was $3 \%$. The patients of higher BMI, DM, age, posteriolateral fusion, female predicted higher incidence of unplanned reoperations. The spine surgeons maybe need to pay more attention in their preoperative training and in improving surgical technique which could reduce the reoperation rate. The spine surgeons maybe need more attention in their preoperative training and in improving surgical technique level of the treatment of with DLS. However, we want to declare that there existed several limitations in this study. First, the study didn't consider the distribution of one-level or two-level DLS in each group which was associated with bias. Second, the retrospective nature of the small-sample study may be associated with bias. In the future, the prospective, randomized studies with long-term follow-up periods are needed. 


\section{Declarations}

Abbreviations PLF: posterior lumbar fusion; DLS: degenerative lumbar spondylolisthesis; BMI: body mass index; DM: diabetes mellitus; DLD: degenerative lumbar diseases; MDT: multiple disciplinary team Availability of data and materials The datasets generated and/or analysed during the current study are not publicly available due to the data is confidential patient data but are available from the corresponding author on reasonable request. Consent for publication Not applicable. Ethics approval and consent to participate The Institutional Review Board of the First Affiliated Hospital of Chongqing Medical University approved this study. Competing interests The authors declare that they have no competing interests. Funding The authors declare no sources of funding. Author details 1Department of Orthopedic Surgery, The First Affiliated Hospital of Chongqing Medical University, Chongqing, China 2Department of Pain Management, The First Affiliated Hospital of Chongqing Medical University, Chongqing, China Authors' contributions Contributions: (I) conception and design: WYZ, XJL2; (II) administrative support: XJL1, ZXQ; (III) provision of study materials or patients: WYZ, XJL2, TJH, ZXQ; (IV) collection and assembly of data: WYZ and XJL1; $(V)$ data analysis and interpretation: WYZ ,TJH, and XJL1. All authors read and approved the final manuscript. Acknowledgements Not applicable.

\section{References}

1. Matz PG, Meagher RJ, Lamer T, et al. Guideline summary review: an evidence-based clinical guideline for the diagnosis and treatment of degenerative lumbar spondylolisthesis. Spine J. 2016;16(3):439-48.

2. Jacobsen S, Sonne-Holm S, Rovsing H,et al.Degenerative lumbar spondylolisthesis: an epidemiological perspective: the Copenhagen Osteoarthritis Study. Spine (Phila Pa1976). 2007;32(1):120-5.

3. Sclafani JA, Constantin A, Ho PS, et al. Descriptive analysis of spinal neuroaxial injections, surgical interventions, and physical therapy utilization for degenerative lumbar spondylolisthesis within medicare beneficiaries from 2000 to 2011. Spine (Phila Pa 1976). 2017;42(4):240-6.

4. Gerling MC, Leven D, Passias PG, et al. Risks factors for reoperation in patients treated surgically for degenerative spondylolisthesis: a subanalysis of the 8 year data from the SPORT trial. Spine (Phila Pa 1976). 2017,42(20):1559-1569.

5. Forsth P, Olafsson G, Carlsson T, et al. A randomized, controlled trial of fusion surgery for lumbar spinal stenosis. N Engl J Med. 2016;374(15):1413-23. 
6. Weinstein JN, Lurie JD, Tosteson TD, et al. Surgical versus nonsurgical treatment for lumbar degenerative spondylolisthesis. N Engl J Med. 2007;356(22): 2257-70.

7. Ghogawala Z, Dziura J, Butler WE, et al. Laminectomy plus fusion versus laminectomy alone for lumbar spondylolisthesis. N Engl J Med. 2016;374(15):1424-34.

8.Challier V, Boissiere L, Obeid I, et al. One-level lumbar degenerative spondylolisthesis and posterior approach: is transforaminal lateral interbody fusion mandatory?A randomized controlled trial with 2-year follow-up. Spine(Phila Pa 1976). 2017;42(8):531-9.

9.Isaacs RE, Sembrano JN, Tohmeh AG, Group SDS. Two-year comparative outcomes of MIS lateral and MIS transforaminal interbody fusion in the treatment of degenerative spondylolisthesis. Part II: radiographic findings. Spine (Phila Pa 1976). 2016;41(Suppl 8):S133-44.

10.Sembrano JN, Tohmeh A, Isaacs R, Group SDS. Two-year comparative outcomes of MIS lateral and MIS transforaminal interbody fusion in the treatment of degenerative spondylolisthesis. Part I: clinical findings. Spine (Phila Pa 1976). 2016;41(Suppl 8):S12332.

11. Matsunaga S, Ijiri K, Hayashi K. Nonsurgically managed patients with degenerative spondylolisthesis: a 10- to 18-year follow-up study. J Neurosurg. 2000;93(2 Suppl):194-8. 12. Pearson AM, Lurie JD, Blood EA, et al. Spine patient outcomes research trial: radiographic predictors of clinical outcomes after operative or nonoperative treatment of degenerative spondylolisthesis. Spine (Phila Pa 1976).2008;33(25):2759-66.

13.Musluman AM, Cansever T,et al. Midterm outcome after a microsurgical unilateral approach for bilateral decompression of lumbar degenerative spondylolisthesis. J Neurosurg Spine. 2012;16(1):68-76.

14. Aihara T, Toyone T, Aoki Y, et al. Surgical management of degenerative lumbar spondylolisthesis: a comparative study of outcomes following decompression with fusion and microendoscopic decompression.J Musculoskelet Res. 2012;15(4):1250020.

15. Inui $T$, Murakami M, Nagao N, et al. Lumbar degenerative spondylolisthesis: changes in surgical indications and comparison of instrumented fusion with two surgical decompression procedures. Spine (Phila Pa 1976). 2017;42(1):E15-24. 
16. Ahmad S, Hamad A, Bhalla A, et al. The outcome of decompression alone for lumbar spinal stenosis with degenerative spondylolisthesis. Eur Spine J. 2017;26(2):414-9.

17. Mardjetko SM, Connolly PJ, Shott S. Degenerative lumbar spondylolisthesis. A metaanalysis of literature 1970-1993. Spine(Phila Pa 1976). 1994;19(20 Suppl):2256S-65S. 18.Ghogawala Z, Benzel EC, Amin-Hanjani S, et al. Prospective outcomes evaluation after decompression with or without instrumented fusion for lumbar stenosis and degenerative Grade I spondylolisthesis. J Neurosurg Spine 2004;1:267-72.

19.Blumenthal C, Curran J, Benzel EC, et al. Radiographic predictors of delayed instability following decompression without fusion for degenerative grade I lumbar spondylolisthesis. J Neurosurg Spine 2013;18:340-6.

20. Martin CR, Gruszczynski AT, Braunsfurth HA, et al. The surgical management of degenerative lumbar spondylolisthesis: a systematic review. Spine (Phila Pa 1976).2007;32(16):1791-8.

21. Rousseau MA, Lazennec JY, Bass EC, et al. Predictors of outcomes after posterior decompression and fusion in degenerative spondylolisthesis. Eur Spine J. 2005;14(1):55-60. 22.Shunsuke Sato, Mitsuru Yagi, Masayoshi Machida et al.Reoperation rate and risk factors of elective spinal surgery for degenerative spondylolisthesis: minimum 5-year follow-up .The Spine Journal, 2015,15:1536-1544 .

23.Liu J-M, Deng H-L, Peng A-F, et al. Unplanned reoperation of lumbar spinal surgery during the primary admission. Spine (Phila Pa 1976) 2016;41:1279-83 .

24.Núñez-Pereira S1,Pellisé F1,Rodríguez-Pardo D, et al. Implant survival after deep infection of an instrumented spinal fusion. Bone Joint J 2013;95-B:1121-6.

25.Kim CH, Chung CK, Park CS, et al. Reoperation rate after surgery for lumbar spinal stenosis without spondylolisthesis: a nationwide cohort study. Spine J 2013;13:1230-7. 26.Schuster JM, Rechtine G, Norvell DC, et al. The influence of perioperative risk factors and therapeutic interventions on infec- tion rates after spine surgery: a systematic review. Spine (Phila Pa 1976) 2010;35:S125-37.

27.Deyo RA. Trends, major medical complications, and charges associated with surgery for lumbar spinal stenosis in older adults. JAMA 2010;303:1259-65. 
28. Okuda S, Iwasaki M, Miyauchi A, et al. Risk factors for adjacent segment degeneration after PLIF. Spine 2004;29:1535-40.

\section{Figures}

\section{Figure 1}

L4 DLS of a 65-year-old woman (BMI 32.3) treated with L4 laminectomy and posteriolateral fusion. Because of leg pain, she had revision (AB) Postoperative X-ray showed the screw displacement. (C) Postoperative X-ray showed the screw was corrected and the pain improved. (D) L4-L5 disc height narrowing and the posteriolateral fusion was observed at the 48 months' follow-up.

\section{Figure 2}

L4 DLS of a 72-year-old man treated with L5 laminectomy and posteriolateral fusion. (AB) Postoperative X-ray was normal. (C) The X-ray showed the screws displacement and fracture at the 36 months' followup.

要

\section{Figure 3}

L5 DLS of a 75-year-old woman treated with L5 laminectomy and intervertebral interbody fusion. (AB) Postoperative X-ray was normal. (C) The X-ray showed the screws displacement and bone union at the 52-months follow-up. 


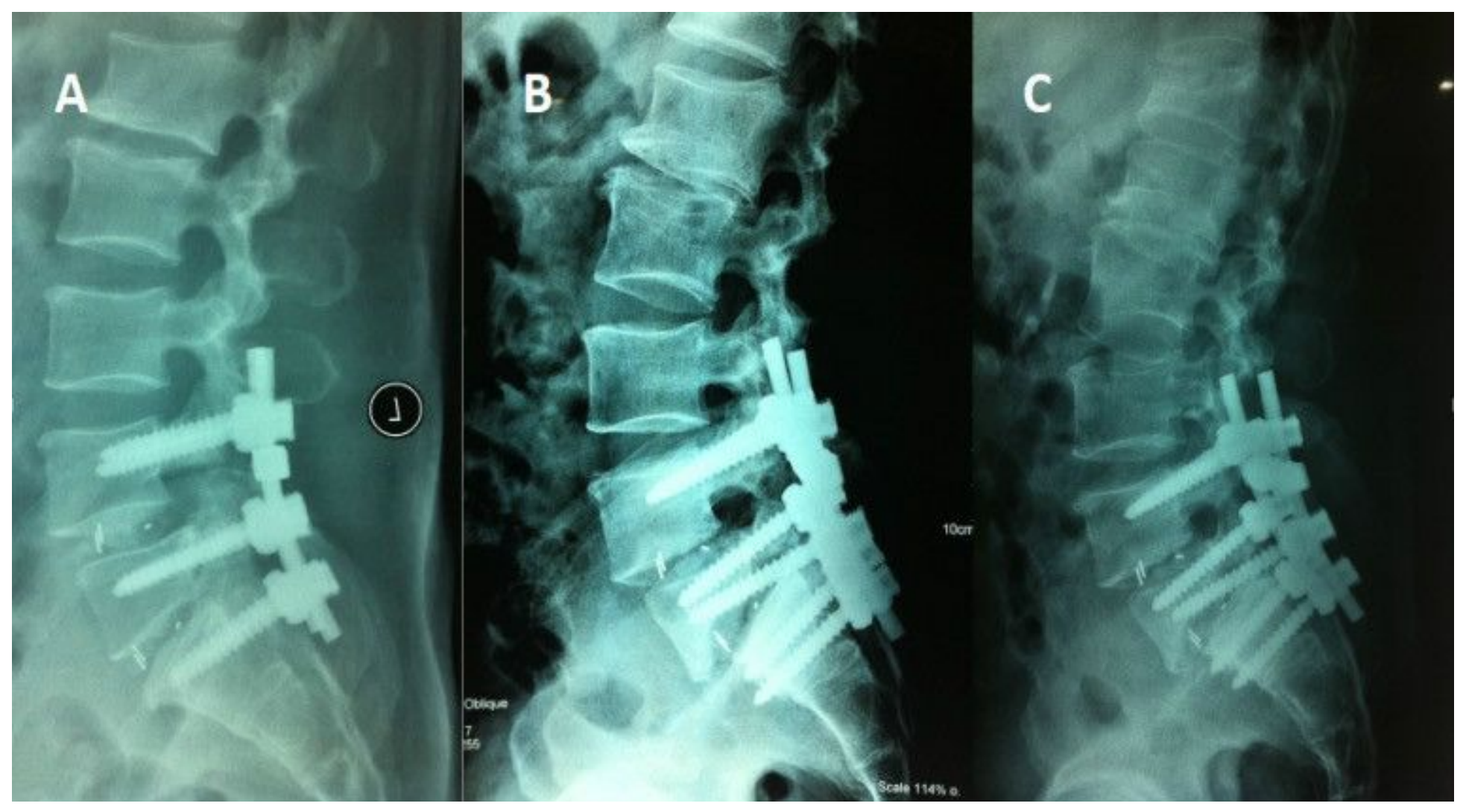

Figure 4

L4 and L5 DLS of a 75-year-old woman treated with L4/5 laminectomy and intervertebral interbody fusion(L4-5, L5-S1). (A囚The postoperative X-ray was normal. (B) The X-ray showed the bone union and screw displacement, the patient refused the revision surgery at the 12 months' follow-up. (C) The X-ray showed the screws displacement and bone union worsen at the 2-years follow-up. The patient suffered leg pain and she accepted the reoperation. 